\title{
DINÁMICA RECIENTE DE LA POBLACIÓN EN LA COMARCA DE OVIEDO (1970-2011)
}

\author{
Ícaro Obeso Muñiz \\ Departamento de Geografía. \\ Facultad de Filosofía y Letras. Universidad de Oviedo \\ C/ Amparo Pedregal s/n, 33011, Oviedo \\ obesoicaro@uniovi.es
}

\begin{abstract}
Resumen: En las últimas cuatro décadas la dinámica de la población asturiana se ha caracterizado por la concentración en su área central. En este contexto, la comarca de Oviedo ha incrementado notablemente su contingente demográfico. A partir de los datos desagregados disponibles más cercanos al poblamiento, las entidades singulares de población, se analiza el patrón espacial y se determinan las fases en las que se produjeron estos cambios. La distribución espacial del fenómeno muestra que las entidades que han experimentado importantes crecimientos se sitúan en las inmediaciones de Oviedo y en los márgenes de los principales ejes de comunicaciones. Por el contrario, las entidades que han sufrido un declive demográfico más acusado se localizan en entornos de marcado carácter rural y en áreas afectadas por el cese de las actividades industriales y mineras.
\end{abstract}

Palabras clave: Oviedo, Asturias, entidades singulares, distribución espacial.

Abstract: In the last four decades, the population dynamics in Asturias has been characterised by the concentration of population in its central area. By focusing on singular population entities - the closest data to settlement, this article analyses the spatial pattern of these dynamics and determines the phases in which these changes have occurred in the Oviedo region. The spatial distribution of this phenomenon shows that the entities that have experienced a significant growth of population are located in the surrounding area of Oviedo and in the margins of the communication axes. On the other hand, the entities that have suffered a sharp demographic decline are located in markedly rural areas and also those affected by the end of mining and industrial activity.

Keywords: Oviedo, Asturias, singular entities, spatial distribution. 


\section{Introducción}

Los paisajes de las principales regiones urbanas contemporáneas se caracterizan por su alto grado de antropización, ya sean estos definidos como urbanos, suburbanos, rururbanos o periurbanos. La acción humana, bien por acción u omisión, ha producido importantes cambios a lo largo del tiempo tanto en la estructura como en la fisonomía. A este respecto, el estudio de la dinámica de la población resulta esencial para comprender las alteraciones recientes. En definitiva, los paisajes muy antropizados tienen en la sociedad que los habita su principal fuerza transformadora; dicho de otro modo, las sociedades desempeñan una labor esencial en su construcción histórica.

La comarca de Oviedo es un espacio enteramente humanizado desde hace siglos al igual que el resto de la España Atlántica (García Fernández, 1975) que se ha caracterizado, en las últimas décadas, por la alteración del modelo de construcción histórica de su paisaje, debido, en gran medida, a los profundos cambios socioeconómicos acontecidos cuyas implicaciones territoriales son más notables en las áreas más urbanizadas, como el área central de Asturias. En referencia a la evolución, el reparto espacial y las características estructurales de la población asturiana, Berta López Fernández (1992) sostiene que el devenir de una sociedad no sólo origina modificaciones, sino que también repercute en la organización del espacio en que ocurren tales transformaciones. Todas estas alteraciones han dado lugar a paisajes difusos, de compleja definición, que no encajan en las habituales clasificaciones entre lo rural y lo urbano, puesto que presentan rasgos propios de ambas tipologías.

La comarca ha experimentado en las últimas décadas importantes transformaciones paisajísticas (Fernández García y Herrán Alonso, 2013). La inauguración en 1976 de la autovía A-66, junto con la construcción de los primeros polígonos industriales situados en el entorno de Oviedo, fueron los primeros elementos novedosos en un paisaje rural que aún se mantenía activo pero que presentaba síntomas de un abandono incipiente (Herrán Alonso, 2002). A partir de entonces se ha ido rompiendo de manera progresiva e ininterrumpida la tradicional dicotomía entre lo rural y lo urbano, cuyas principales consecuencias han sido, entre otras: la mezcla de usos del suelo, el incremento de la vivienda unifamiliar aislada (Herrán Alonso, 2008), el abandono de las actividades agroganaderas (Morán Alonso et al., 2017), la proliferación de autovías y carreteras y el consecuente aumento de la accesibilidad y de la movilidad diaria; y finalmente, la difusión sobre el medio rural de funciones netamente urbanas tales como la educativa, la comercial o la del ocio. En suma, hemos asistido a un proceso de intensa transformación territorial, que diferentes autores han dado en llamar difusión urbana (Indovina, 1990; Delgado Viñas, 1999; Fernández García, 2003).

Esta transformación territorial tiene una dimensión visible que es aquella que implica la aparición de nuevos elementos sobre el paisaje: equipamientos, infraestructu- 
ras o el avance de la urbanización en el borde de los núcleos de población más importantes. Otra componente de la fisonomía del paisaje, aunque menos estudiada, es la de la desaparición, en la que destacan los procesos de desuso y abandono, que permanecen en estado ruinoso ante nuestros ojos y tras su definitiva desaparición, solamente viven en nuestra memoria y en los archivos de fotografías históricas.

Sin embargo, los cambios territoriales recientes también tienen ciertas características que son invisibles, entre las que se incluyen las permutas de la propiedad, la accesibilidad a un determinado lugar o los ritmos demográficos. Por ser especialmente lentos y difíciles de percibir, estos cambios demográficos son estudiados en este artículo desde una perspectiva histórica, pero también espacial, con el objetivo de explicar cómo ha evolucionado la población de la comarca de Oviedo entre 1970 y la actualidad, en qué medida se pueden establecer fases que permitan una organización temporal de los cambios y qué implicaciones territoriales ha supuesto esta dinámica demográfica reciente.

En el conjunto de las contribuciones académicas referidas al análisis de la población en entornos comarcales y metropolitanos no suelen emplearse las entidades de población sino que la gran mayoría de este tipo de estudios se centran, bien en el nivel municipal, bien en temáticas concretas como los movimientos pendulares o la población vinculada. Algunos estudios de geografía de la población utilizan otro nivel inferior al municipal, el de las parroquias. Tanto en la comunidad autónoma gallega como en la asturiana se conservan las parroquias, entidades colectivas que no tienen naturaleza política ni administrativa, pero de utilidad con fines estadísticos. La parroquia responde a un nivel inferior al municipal y se forma por la agrupación de entidades singulares de población.

La recogida de datos originales provenientes de fuentes estadísticas impresas como los Nomenclátores de 1970, 1981 y 1991 junto con la explotación de la fuente disponible en formato electrónico, para periodos censales posteriores, en la web del INE permiten la georreferenciación de las entidades, y por tanto, el estudio, con mayor grado de detalle, de la dinámica espaciotemporal de la población en un determinado ámbito.

En el presente artículo se aborda el análisis de la dinámica de las entidades de población desde 1970, fecha en la que comienza a romperse la tradicional dicotomía urbano-rural en la comarca de Oviedo dando lugar a un paisaje fuertemente antropizado. Con el estudio detallado de la dinámica de la población se pretende determinar qué entidades han experimentado procesos de transformación más intensos, sean estos de crecimiento o de declive demográfico. Al mismo tiempo, se estudian los ritmos de cambio y se identifican las principales fases. Finalmente, y dado que el crecimiento demográfico no se distribuye de modo homogéneo, se pone el foco en la perspectiva espacial con el objetivo de clasificar diferentes unidades espaciales en la comarca de Oviedo en función de su comportamiento demográfico, que servirá de base para futuras investigaciones sobre los procesos de cambio en los usos del suelo, la difusión urbana y las transformaciones paisajísticas acontecidas en las últimas décadas. 


\subsection{La dinámica reciente de la población en las áreas urbanas a diferentes escalas}

Como constata el informe de la urbanización mundial (UN, 2014), el siglo XX se ha caracterizado por el rápido crecimiento de la población urbana en el mundo. Según los datos recogidos por el organismo internacional, en 2007 se produjo un cambio de paradigma en la población mundial ya que, por primera vez en la historia, la población urbana superaba a la rural y la separación entre ambas se incrementa cada año desde entonces. Dentro de las diferentes tipologías de ciudad definidas por el informe, en las ciudades pequeñas y medianas, es decir, las de menos de 500.000 habitantes, vive más de la mitad de la población mundial. Esta tendencia se refleja en el comportamiento reciente del continente europeo que pasó del 59,7\% en 1970 al 73,9\% en 2015. Este aumento de la población urbana en Europa ha implicado profundas transformaciones paisajísticas en las áreas más urbanizadas del continente, como ha sintetizado Antrop (2004), mientras que otros autores como Champion (2001), Cheshire (1995), Van der Bergh et al. (1982) y Klaassen et al. (1981) han puesto el foco en la identificación de las fases del proceso.

La evolución de la población española en el último siglo ha estado marcada por drásticos cambios, en especial, por el aumento de la población urbana y por el éxodo rural (Puyol, 1999; Arroyo Pérez, 2003; López Fernández, 2010). Las cifras del informe de la ONU (UN, 2013) muestran que el aumento de la población urbana en España ha sido más acelerado que en el resto del continente, ya que desde 1970 a la 2015, ha pasado del 66\% al 78,3. En relación al fenómeno de concentración de la población dentro de las grandes regiones urbanas se han publicado numerosos trabajos de investigación que superan la escala municipal y abordan ámbitos comarcales, a veces, denominados también metropolitanos. Sirvan como ejemplos, la influencia de Madrid en las comarcas castellano-manchegas, que ha sido estudiada por Pozo Rivera y Rodríguez Moya (2006); la evolución demográfica reciente de la población andaluza, por Fernández Salinas (2001); o los estudios de síntesis de la población en las áreas metropolitanas españolas que demuestran pautas y comportamientos homogéneos incluso a escala municipal (De Cos Guerra, 2007).

Entre los estudios básicos y pioneros acerca de la evolución histórica de la población asturiana se encuentran los realizados por Criado y Pérez (1975) y el de Pérez González, Fernández García y Morales Matos (1983). Ambos trabajos fueron posibles, en gran medida gracias a la elaboración previa de la división parroquial de Asturias realizada por Francisco Quirós Linares (1982). Desde entonces, los estudios de la población asturiana alcanzaron un mayor detalle, como demuestra el trabajo sobre la evolución de la población entre 1900 y 1981 a escala parroquial (Fernández García y Maceda Rubio, 1988) o más recientemente, en lo que concierne al declive demográfico entre el 2000 y 2014 (López Fernández, 2016), que aporta una brillante prognosis sobre el despoblamiento. Ambos estudios ponen de manifiesto que, a pesar de la concentración de la población en el área central asturiana, no todas las parroquias del centro de la comunidad autónoma presentan crecimientos demográficos sino que también existen entidades colec- 
tivas que se caracterizan por un continuado declive demográfico, de modo similar a los espacios rurales de las alas o a las zonas que han sufrido los efectos de la crisis industrial y minera. Posteriormente, este tipo de trabajos fueron actualizados en diferentes obras colectivas, en su mayoría atlas geográficos, como el Atlas social de las mujeres asturianas (1994), Atlas Escolar de Asturias (2005) o el Atlas Temático de España (2010).

Desde un punto de vista urbano, también se han llevado a cabo estudios de población en Langreo (Fernández García, 1985), Gijón (López Fernández, 1988) y Oviedo (Obeso Muñiz y Cortizo Álvarez, 2016). Sin embargo, la perspectiva comarcal no ha sido tan utilizada y, cuando se hace referencia a ella, se limita a la escala parroquial o a un sector de actividad concreto. La contribución más parecida, a escala parroquial, fue la que estudia la dinámica reciente de la comarca de Avilés (Fernández Soto, 2014). Desde un punto de vista más explicativo que cuantitativo e integrado en publicaciones colectivas, la población de la comarca de Oviedo ha sido objeto de estudio en diversos capítulos de la Geografía de Asturias (1992) y en obras que vinculan los indicadores socioeconómicos a lo territorial (Fernández García, 2007).

\subsection{La dinámica reciente de la población asturiana}

Pese a que este artículo pone el foco en la evolución reciente, se considera oportuno apuntar algunas de claves sobre la historia demográfica asturiana. Durante el siglo XX y los inicios de la centuria siguiente, Asturias se caracteriza por un crecimiento continuado de su población hasta 1981, año en el que se alcanza el máximo histórico; 1.129.556 habitantes. Desde entonces, la tendencia ha sido regresiva (INE, 2012). Este hecho se explica por la relación que han tenido la industrialización y las migraciones en Asturias desde que se elaborase el primer censo en 1857 hasta la actualidad. Quizás, también sea esta una de las razones por las que la actual situación despierta tantas alarmas y polémicas en torno al paulatino descenso demográfico, que no deja de ser una vuelta a la situación precedente a la extracción del carbón y la industrialización, procesos que atrajeron mano de obra e impulsaron la natalidad en la región.

Esta industrialización, encontró su lugar preferente en el área central de la región (Fernández García, 1984) y desencadena, en parte, la actual distribución de la población determinada por altas densidades de población en el centro y densidades más bajas en el resto del territorio. En la actualidad, en las tres principales ciudades asturianas (Gijón, Oviedo y Avilés) viven más de la mitad de sus habitantes, proporción que asciende al 75\% si tenemos en cuenta todos los municipios del centro de Asturias.

Entre 1950 y 1981, esta concentración de la población propició el crecimiento de los núcleos de población, pero también la construcción de infraestructuras y equipamientos, así como un considerable aumento de la motorización. Conviene mencionar que no todas las transformaciones del área central asturiana se asocian a la adición o 
superposición de nuevos elementos y funcionalidades, sino que también se han desencadenado procesos de abandono y desaparición, especialmente en lo que concierne a la minería, la industria pesada y las actividades propias del sector primario.

Desde 1981, se viene constatando un ligero descenso demográfico en Asturias. El envejecimiento de la población y la constante emigración de adultos jóvenes refuerzan esta tendencia que se agrava especialmente en la comarca suroccidental, en las áreas de montaña y en aquellos lugares en los que las sucesivas crisis económicas han desmantelado tejidos productivos muy especializados. En los concejos de Langreo y Mieres el declive demográfico comenzó en 1960, repitiéndose dicha tendencia en todos los municipios de las Cuencas Mineras.

Al decrecimiento se le añadió un saldo migratorio también negativo, todo ello junto a los trascendentales cambios políticos, culturales y socioeconómicos. Todo lo cual produjo que en 1986 se iniciase una etapa de comportamiento demográfico inédita en Asturias, como refleja el gráfico (Fig. 1) actualizado a partir del original elaborado por Criado y Pérez (1975).

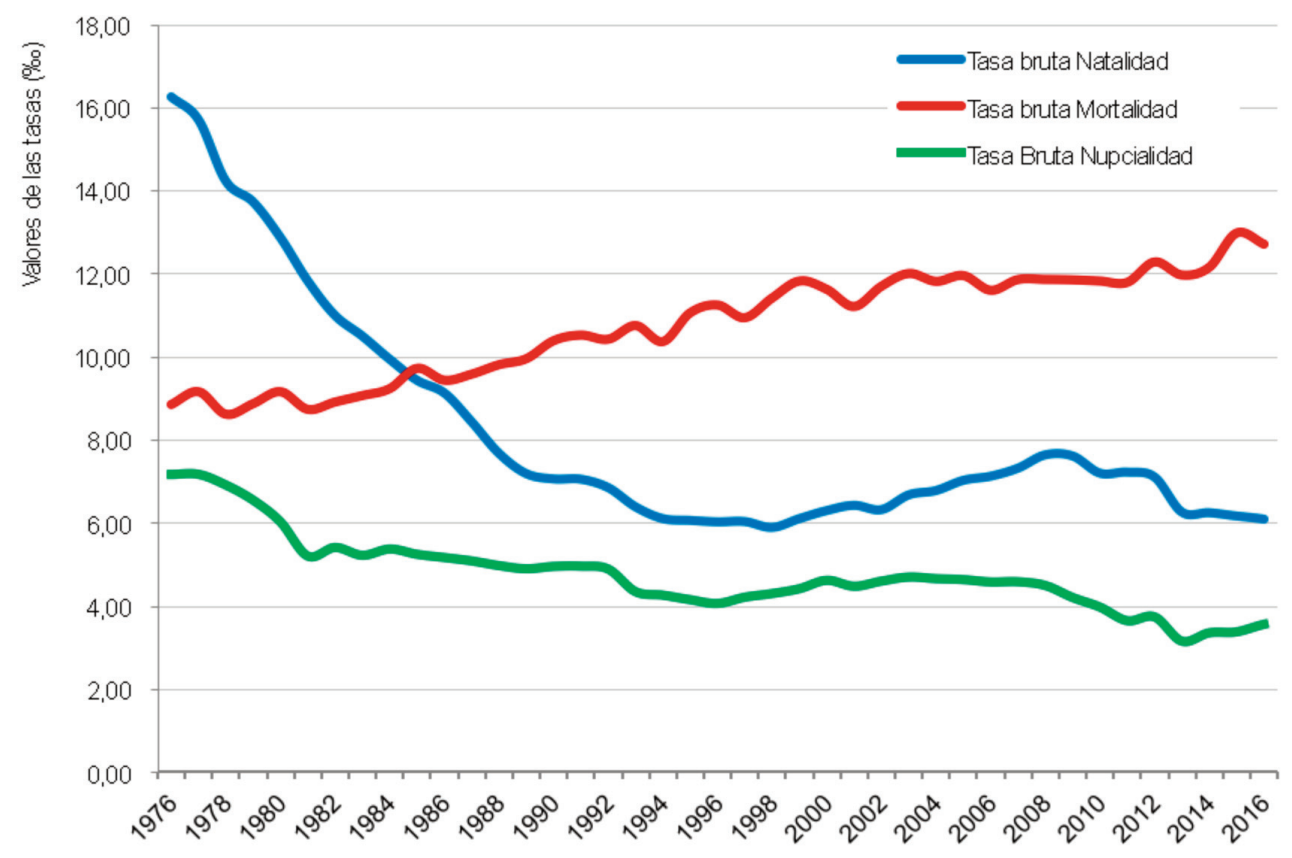

Figura 1. Evolución de la tasas brutas de natalidad, mortalidad y nupcialidad en Asturias entre 1976 y 2016.

Fuente: Elaboración propia a partir de la actualización del gráfico original elaborado por Criado y Pérez (1975). 
No obstante, la concentración de la población no se debe exclusivamente al peso de las tres principales ciudades, sino también al resto de municipios que conforman el área central. Así, los concejos de Siero, Llanera y Noreña han tenido un notable crecimiento demográfico lo que constituye una evidencia de que este espacio periurbano de la comarca de Oviedo ha sufrido importantes transformaciones tanto demográficas como paisajísticas. Por tanto, la comarca de Oviedo se caracteriza por una dinámica demográfica favorable como reflejan las cifras del Padrón Municipal, la población de la comarca se incrementó en un 41\% entre 1970 y 2015 cuando alcanzó los 297.122 habitantes.

\section{2. Área de estudio}

La comarca de Oviedo está emplazada en el norte de la Península Ibérica (aprox. $43.4^{\circ}$ N, $5.8^{\circ}$ O) y está conformada por los municipios de Llanera, Noreña, Oviedo, Las Regueras, Ribera de Arriba y Siero que suman $598 \mathrm{~km}^{2}$. La comarca no responde a ninguna entidad política o administrativa ni tiene ningún tipo de uso en la desactualizada ordenación territorial asturiana y tampoco se utiliza como unidad estadística. Esta comarca, por tanto, ha sido delimitada a partir de criterios paisajísticos y funcionales con el fin de abordar el análisis de las transformaciones paisajísticas recientes. Dado que las parroquias y los límites municipales en Asturias se corresponden normalmente con accidentes geográficos, el hecho de definir el perímetro de una comarca ya implica cierta analogía con una unidad paisajística. De todas formas, los criterios paisajísticos que definen la comarca son varios: una suave topografía rodeada por las sierras prelitorales que limitan septentrionalmente los municipios de Las Regueras, Llanera y Siero y que actúan al mismo tiempo como límite perceptual al dibujar una orla de colinas de escasa altitud, de modo similar sucede en los bordes meridional y occidental. Hacia el este, la depresión prelitoral continúa estrechándose más allá del municipio sierense. En definitiva, en la comarca se encuentra la mayor superficie de topografía llana de toda la región ya que los suelos que tienen una pendiente inferior al 3\% suponen unos $100 \mathrm{~km}^{2}$.

En cuanto a criterios funcionales se han tenido en cuenta las actividades y los usos del suelo. En toda la comarca se aprecia la proximidad a Oviedo y el papel de las infraestructuras de transporte y comunicación como elementos estructurantes de este espacio. Las funciones tradicionalmente urbanas como la industrial, la comercial y la residencial aparecen dispersas en el territorio y en menor medida otras como la hostelera, la educativa o la del ocio. Esta difusión funcional implica una alta heterogeneidad de los usos del suelo que conlleva la presencia de incompatibilidades, molestias y la presencia de suelos sin uso debido, principalmente, a una Ordenación del Territorio muy laxa y desactualizada.

La figura 2 refleja la organización administrativa de la comarca, en la que se representan los cinco municipios y los límites parroquiales. En suma, la comarca de Oviedo 


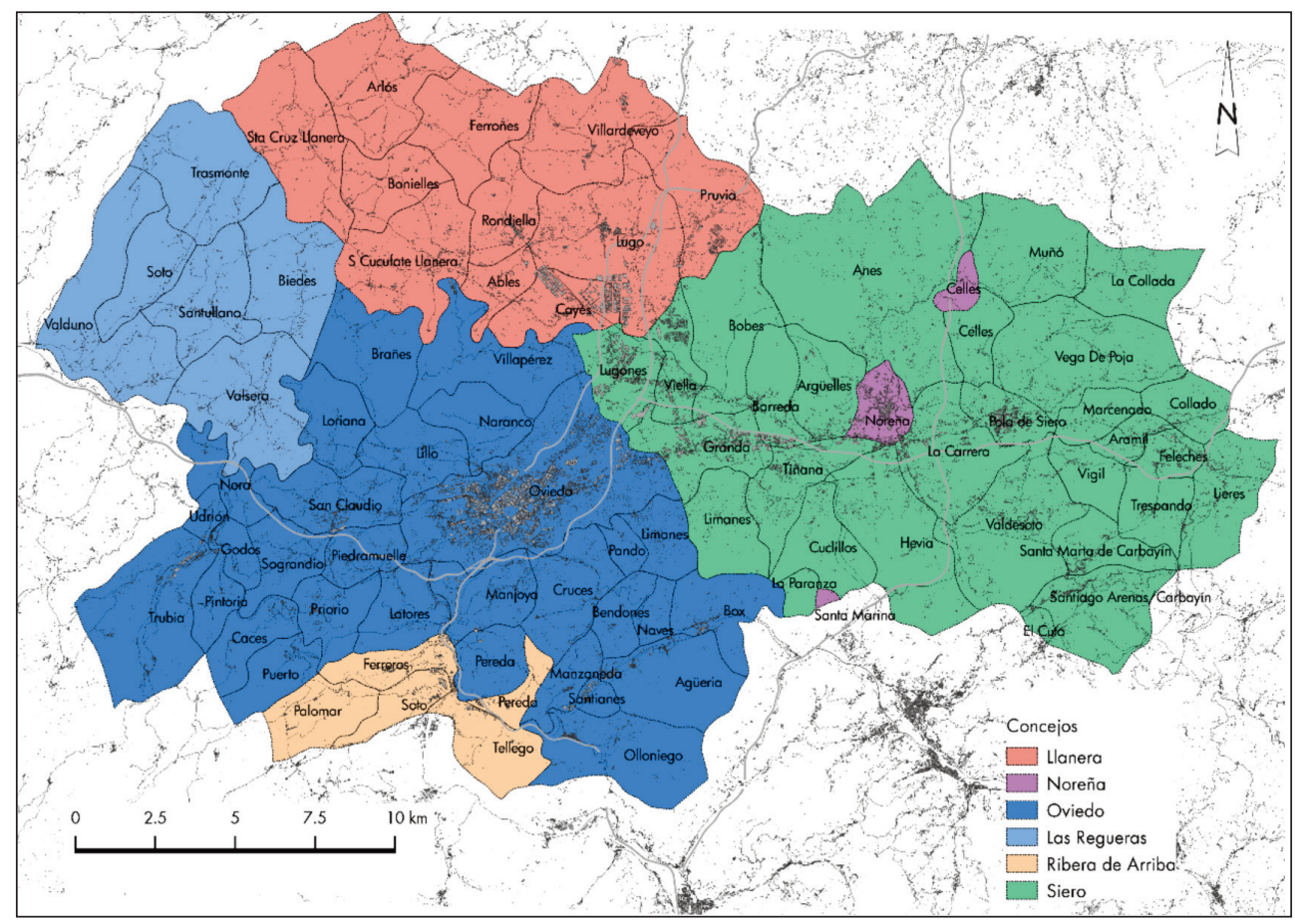

Figura 2. La comarca de Oviedo. Municipios que la conforman y división parroquial.

Fuente: Elaboración propia a partir de la división parroquial efectuada por Quirós Linares (1982) y digitalizada por José Ramón Fernández Prieto, ambos profesores del Departamento de Geografía de la Universidad de Oviedo.

se compone de 84 parroquias o entidades colectivas y 681 entidades singulares. Como se aprecia en la imagen la difusión urbana de la comarca se caracteriza por un núcleo urbano principal, Oviedo, del que irradian diferentes ejes articulados por las infraestructuras y entorno a los que se sitúa la mayor parte del espacio construido, de tal forma que en dirección a Gjón y Avilés se genera un corredor formado por Lugones, Posada y Lugo de Llanera, hacia Santander un nuevo eje que cruza Colloto, Granda, Noreña y Pola de Siero, siguiendo la depresión prelitoral. Existe una difusión de la función residencial de baja densidad que se superpone al tradicional poblamiento de la vertiente cantábrica, es decir, de tipo disperso intercalar por agrupación de aldeas, quintanas y praderías, que se localizan en los lugares de topografía más favorable, mejor soleamiento y máxima accesibilidad. Finalmente, el valle del Trubia y del Nalón entre Olloniego y Tudela-Veguín, junto a las parroquias del Sur de Siero (Arenas, Carbayín y Lieres) se localizan los principales núcleos vinculados a la tradición industrial y minera. 
En las parroquias de Oviedo, las sierenses de Lugones, Viella, Bobes, Granda y Argüelles, junto con las de Pruvia, Cayés y Lugo en Llanera, se concentran los principales elementos propios de un paisaje periurbano caracterizado por la heterogeneidad de usos del suelo, la fragmentación, la máxima accesibilidad y concentración de infraestructuras y actividades y, en definitiva, aquí confluyen importantes enlaces de autovías, con varios polígonos industriales, equipamientos comerciales de ámbito regional y promociones de vivienda de variadas tipologías.

\section{Datos y metodología}

Los datos de la población organizados por entidades son recogidos por el Nomenclátor que es una fuente estadística elaborada por el Instituto Nacional de Estadística (INE) a partir de los datos del Padrón Continuo por Unidad Poblacional que mantienen y actualizan los municipios. Existen Nomenclátores por cada año censal y, a partir del 2000, esta fuente se actualiza con una periodicidad anual. En este trabajo se han tenido en cuenta los nomenclátores publicados en 1970, 1981 y 1991, que están disponibles, sólo en papel y los digitalizados que proporciona el INE en su web desde el año 2000.

En una primera fase se obtuvieron los datos para los años censales 2001 y 2011 y se construyó una tabla en un programa de hojas de cálculo a la que se fueron añadiendo posteriormente los datos extraídos de los Nomenclátores originales, publicados en papel para los años anteriores: 1991, 1981 y 1970. De esta manera, se ha mantenido la coherencia de los códigos y de la organización de las entidades por parroquia. Naturalmente, desde 1970 hasta la actualidad ha habido cambios: reestructuración de límites parroquiales, cambios de nombre, recuperación de la toponimia tradicional, aparición de nuevas entidades, etc. Las alteraciones no han sido tan abundantes como para comprometer la viabilidad del análisis. Producto de la modernización del INE, de las transformaciones paisajísticas recientes y de los cambios demográficos acontecidos en la comarca, a partir de 1991 se constata un aumento del número de entidades singulares de población.

El tratamiento gráfico de la información se ha llevado a cabo mediante la utilización de un sistema de información geográfica libre que permite la coordinación entre la información estadística tabulada y la base cartográfica. La información cartográfica de referencia ha sido obtenida a partir de diversas fuentes: Instituto Geográfico Nacional (IGN), Sistema de Información Territorial del Principado de Asturias (SITPA) y el mapa parroquial de Asturias elaborado por Francisco Quirós Linares $(1982,1993)$ y digitalizado por José Ramón Fernández Prieto, profesor titular del Departamento de Geografía de la Universidad de Oviedo. 


\section{Resultados}

De acuerdo con las cifras del Padrón Municipal, la población de la comarca en 1970 era de 210.098 habitantes y en el año 2015 ascendía a 297.122, lo que supone un incremento del 41\%. Este hecho pone de manifiesto la importancia que ha tenido la componente antrópica en la dinámica reciente de la comarca de Oviedo.

\subsection{La dinámica demográfica (1970-2011)}

Los datos obtenidos del INE muestran un claro crecimiento sostenido en la comarca desde 1970 hasta 2011, año del último censo disponible. El cuadro 1 revela la paulatina concentración de la población en el área central de la región, puesto que el peso demográfico de la comarca sobre el total de Asturias ha pasado de un 20\% a un 28\%. En términos absolutos se distinguen dos tendencias claramente diferenciadas. Por un lado están los municipios cuya evolución demográfica ha sido positiva, entre los que se encuentran Llanera, Noreña, Oviedo y Siero; mientras que los concejos de Las Regueras y Ribera de Arriba; en el borde sur y occidentales han experimentado pérdida de habitantes. Estas tendencias se mantienen desde 1981, observándose ligeras alteraciones en los censos precedentes en los municipios de Llanera y Ribera de Arriba.

Cuadro 1. Dinámica reciente de la población en la comarca de Oviedo

\begin{tabular}{|l|r|r|r|r|r|}
\hline & 1970 & 1981 & 1991 & \multicolumn{1}{c|}{2001} & \multicolumn{1}{c|}{2011} \\
\hline Llanera & 10.099 & 9.966 & 10.457 & 12.183 & 14.036 \\
Noreña & 3.673 & 4.155 & 4.190 & 4.349 & 5.432 \\
Oviedo & 154.117 & 190.123 & 196.051 & 201.005 & 225.391 \\
Regueras, Las & 3.342 & 2.880 & 2.427 & 2.155 & 1.981 \\
Ribera de Arriba & 2.971 & 2.532 & 2.198 & 1.984 & 1.984 \\
Siero & 35.896 & 40.222 & 44.033 & 48.368 & 52.094 \\
\hline Comarca & 210.098 & 249.878 & 259.356 & 270.044 & 300.918 \\
\hline Asturias & 1.052 .048 & 1.087 .728 & 1.093 .937 & 1.075 .329 & 1.081 .487 \\
\hline \% sobre el total & $20 \%$ & $23 \%$ & $24 \%$ & $25 \%$ & $28 \%$ \\
\hline
\end{tabular}

Datos obtenidos de los censos de población elaborados por el Instituto Nacional de Estadística. 
A pesar de que Asturias alcanzó el máximo de población en 1991 y desde entonces pierde población, el proceso de concentración de la población en su área central no se ha detenido como demuestran las cifras del cuadro. En concreto, el peso de la comarca de Oviedo no ha cesado de aumentar respecto del total regional. Los mayores incrementos se dan entre 1970-1981 y 2001-2011 en gran medida debido a los crecimientos experimentados por el concejo de Oviedo, observándose una fase de crecimientos moderados entre 1981 y 2001. Con mayor detalle la dinámica reciente se representa en el gráfico (Fig. 3).

En términos relativos y de acuerdo con los datos del Padrón, se verifica un incremento poblacional en las últimas décadas, aunque existe un ligero descenso desde el última año censal, 2011, hasta el último padrón consultado, el de 2015. La siguiente figura (Fig. 3) muestra la dinámica regresiva de los municipios de Ribera de Arriba y Las Regueras frente al crecimiento del resto de concejos de la comarca. El gráfico revela que el mayor incremento poblacional ha sido el de Noreña entre 2004 y 2010 cuya dinámica reciente ha sido analizada por Aladino Fernández García (2015). Los municipios de Siero y Oviedo han mantenido un comportamiento similar al del total comarcal, mientras que Llanera vivió una fase de acoplamiento entre 1991 y 2005.

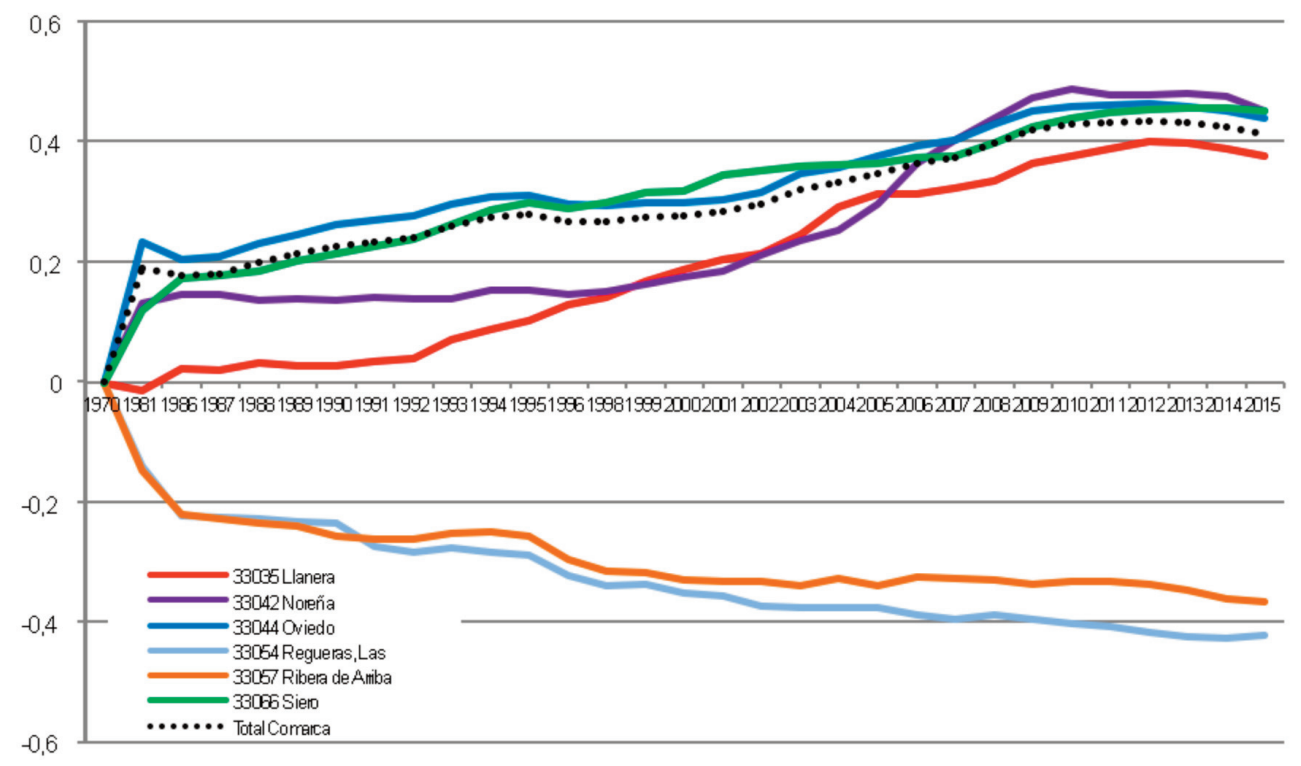

Figura 3. Gráfico comparativo de la evolución demográfica (\%) de los municipios que conforman la comarca de Oviedo. Se ha establecido el valor 0 para 1970.

Fuente: Elaboración propia a partir de datos censales y padronales obtenidos del INE y del SADEI. 
La mayoría de los análisis de la población en ámbitos comarcales o en áreas metropolitanas se limitan al estudio de los datos municipales y no es frecuente el empleo de las entidades de población. Esto se debe a la difícil tarea de analizar un gran conjunto de datos para una entidad supramunicipal; al mismo tiempo que se pone el foco en lo pequeño; es decir las entidades de población (López Fernández, 2016). Las escala empleada facilita el objetivo de transmitir una síntesis que permita extraer conclusiones generales y unos patrones espaciales a la hora de comprender los importantes cambios experimentados en la comarca en las últimas décadas.

\subsection{La distribución espacial de la variación de población}

Los patrones espaciales de cualquier fenómeno geográfico se nos escapan, en muchas ocasiones, por la falta de datos, por la propia autocensura de quien los analiza o bien por una mala elección de la escala. En este caso, se utilizan las entidades de población, las unidades estadísticas más cercanas a la realidad del poblamiento, ya que el juego de escalas es fundamental para comprender cualquier fenómeno representado espacialmente, puesto que la realidad se muestra diferente según la escala utilizada (Lacoste, 1977).

El Nomenclátor, aunque es parco en información, cumple con las expectativas de profundización del análisis tanto en la escala como en la cercanía a la realidad del poblamiento, puesto que permite obtener los datos de las entidades de población, tanto las colectivas denominadas comúnmente parroquias, como las entidades singulares que en función del tamaño abarcan tanto las ciudades y las villas, como los pequeños pueblos y aldeas. La fuente recoge tan sólo la población, desglosada en hombres y mujeres, la altitud y las coordenadas geográficas. Los códigos establecidos por el INE y las coordenadas geográficas han permitido la vinculación de la información alfanumérica con la base cartográfica y, por tanto, la representación cartográfica del fenómeno.

Tras el volcado de los datos recogidos en los diferentes Nomenclátores que van desde 1970 a 2011, se elaboró una tabla con la información alfanumérica de 654 entidades de población. Con el objetivo de obtener una clasificación significativa espacialmente, se calcularon las tasas de crecimiento entre 1970 y 2011 de aquellas entidades de más de cinco habitantes y se comprobó que la comarca tiene un comportamiento demográfico dual. Por un lado, existen 98 entidades cuyo crecimiento es positivo; por otro, 400 en las que ha descendido el número de habitantes y finalmente 12 entidades que han permanecido en una situación de estancamiento. Además este comportamiento tan diferenciado tiene una clara distribución espacial por lo que fueron establecidas diferentes clases a la hora de elaborar una representación cartográfica que pusiera de manifiesto esta realidad. Se elaboró un histograma con el conjunto de datos y se establecieron las categorías de acuerdo con unos umbrales que permitiesen la rápida lectura de la dinámica demográfica reciente: 
- Fuerte crecimiento: crecimientos superiores al 25\%

- Crecimiento moderado: entre 1\% y $25 \%$

- Estancamiento demográfico: entre -1\% y $1 \%$

- Descenso moderado: entre el -1 y -25\%

- Descenso acusado: entre -25\% y $-50 \%$

- Descenso muy acusado: descensos mayores del 50\%

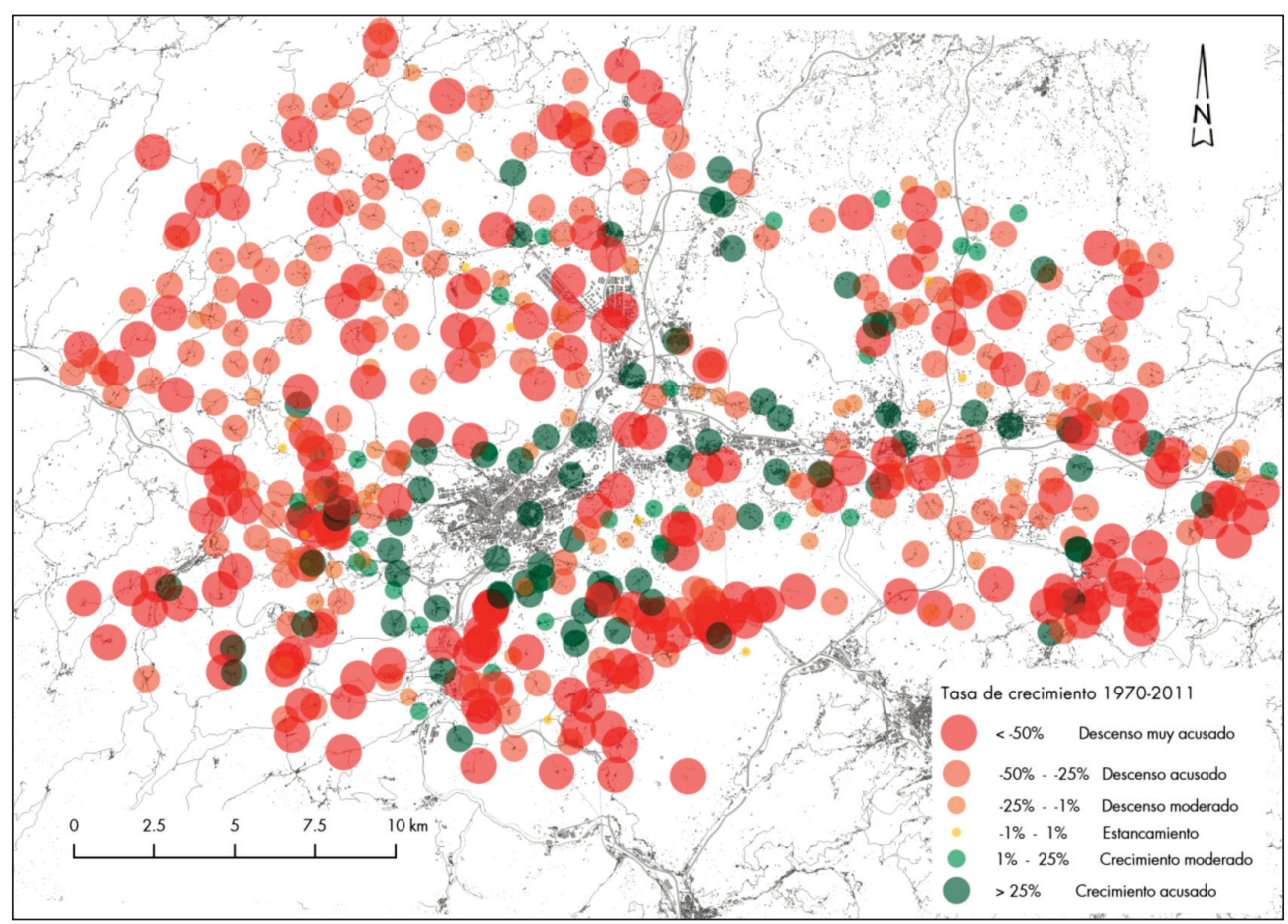

Figura 4. Distribución espacial de las tasas de crecimiento entre 1970 y 2011 en las entidades singulares de población de la comarca de Oviedo.

Fuente: Elaboración propia a partir de la información contenida en los Nomenclátores publicados por el INE.

La clasificación representada en la figura 4 ilustra un patrón espacial muy significativo. De tal forma que aquellas entidades que han tenido un descenso poblacional más acusado se sitúan en los márgenes de la comarca, en zonas con peor accesibilidad tanto en transporte público como en vehículo privado; y se caracterizan por el predominio histórico de las actividades del sector primario y del secundario especializado en minería e industria básica. 
La clasificación y la representación de los datos por umbrales permiten el análisis detallado de los diferentes comportamientos demográficos. Dentro del conjunto de entidades que pierden población se han establecido tres umbrales. El descenso muy acusado, es decir, mayor al 50\% se localiza en entidades pequeñas y remotas, en su mayoría aldeas, pero también en entidades afectadas por procesos de crisis y desindustrialización como son Trubia, Olloniego, La Manjoya, San Claudio, Carbayín y Lieres.

Los descensos acusados y los moderados se distribuyen de forma homogénea en la totalidad del espacio habitado de la comarca, sin observarse ningún patrón espacial significativo. En términos generales, el descenso de población acontece en todos los municipios estudiados. En mayor medida en el de Las Regueras, en la zona septentrional y occidental de Llanera, en la parte oriental de Siero, tanto en las inmediaciones de la Peña Careses, al noreste del concejo, como en los antiguos enclaves mineros de Carbayín y Lieres, al sureste. Mientras que en el municipio de Oviedo, la tendencia regresiva se observa en la vertiente norte del Monte Naranco y también en las parroquias de Nora, Trubia y Godos en el sector suroccidental del concejo, así como en el valle del Nalón entre Olloniego y Tudela-Veguín.

Por el contrario, las entidades que han aumentado su población entre 1970 y 2011 se localizan en los núcleos habitados de una cierta entidad como Noreña y los núcleos sierenses de Lugones, El Berrón y Pola de Siero; y Posada y Lugo en Llanera. La tasa de crecimiento más alta de toda la comarca es la de La Corredoria, antiguo pueblo a los pies de la antigua carretera Oviedo-Gijón que se ha convertido en un barrio de la capital y que actualmente alberga unas 15.000 personas. En el resto del concejo de Oviedo se observan crecimientos moderados y acusados en las entidades que rodean la ciudad, las cuales dibujan una orla o franja periurbana en la que destacan los crecimientos positivos de las entidades situadas en la vertiente soleada del Naranco; las parroquias meridionales de Cruces y Bendones; así como algunas entidades emplazadas al Oeste entre San Claudio y Oviedo. Es en estos espacios del municipio ovetense en los que la producción del espacio residencial de baja densidad ha cobrado mayor protagonismo (Herrán Alonso, 2008).

\subsection{Fases del proceso}

De modo complementario al estudio de la distribución espacial de la dinámica demográfica, resulta interesante conocer las diferentes fases del proceso para poder establecer futuras relaciones entre los períodos de mayor crecimiento poblacional con la antropización del paisaje de la comarca. A pesar del incremento de población en el periodo estudiado, el comportamiento demográfico no ha sido homogéneo en las diferentes fases. Mientras que el crecimiento de la comarca fue del 44\%, el análisis por períodos intercensales demuestra que el crecimiento del contingente demográfico ha sido 
mayor entre 1970 y 1981, cuando el conjunto incrementó su población en un 17\%, seguido de una fase de crecimiento más moderado entre 1981 y 2001. En la última década disponible, esto es, entre 2001 y 2011, la población aumentó un 11\%. En la siguiente tabla se detallan las diferentes fases del proceso:

Cuadro 2. Evolución demográfica de los municipios por períodos intercensales

\begin{tabular}{|l|c|c|c|c|c|}
\hline Municipio & $1970-1981$ & $1981-1991$ & $1991-2001$ & $2001-2011$ & $1970-2011$ \\
\hline Llanera & $-1 \%$ & $5 \%$ & $17 \%$ & $15 \%$ & $39 \%$ \\
Noreña & $15 \%$ & $1 \%$ & $4 \%$ & $25 \%$ & $51 \%$ \\
Oviedo & $21 \%$ & $6 \%$ & $3 \%$ & $12 \%$ & $48 \%$ \\
Las Regueras & $-14 \%$ & $-16 \%$ & $-11 \%$ & $-8 \%$ & $-41 \%$ \\
Ribera de Arriba & $-15 \%$ & $-14 \%$ & $-10 \%$ & $0 \%$ & $-34 \%$ \\
Siero & $11 \%$ & $9 \%$ & $10 \%$ & $8 \%$ & $43 \%$ \\
\hline Total & $17 \%$ & $6 \%$ & $4 \%$ & $11 \%$ & $44 \%$ \\
\hline
\end{tabular}

Fuente: Elaboración propia a partir de los datos de los nomenclátores publicados por el INE.

La variación intercensal muestra las fases del crecimiento de la población en la comarca. Los municipios que han experimentado un crecimiento mayor son los de Noreña, Oviedo, Siero y Llanera, mientras que los concejos de Las Regueras y Ribera de Arriba se caracterizan por un paulatino descenso demográfico. Las fases de mayor crecimiento para cada municipio son diferentes; así, en Llanera la década entre 1991 y 2001 es la que representa un mayor incremento, en Noreña esta fase tuvo lugar en la década posterior, y en Oviedo y Siero aconteció entre 1970 y 1981.

A escala parroquial, el análisis esconde los cambios acontecidos en algunas entidades singulares, como son los casos de las situadas en el entorno de Carbayín y Hevia en Siero, municipio donde se han registrado más cambios. La escala parroquial, dificulta, al mismo tiempo una lectura efectiva de la variación de población entre 1970 y 2011, puesto que tan sólo 19 de las 83 parroquias de la zona de estudio han incrementado su población, lo que enmascara el análisis más preciso efectuado por entidades de población que permite acercarse más a la realidad territorial.

Es significativo, sin embargo, observar el crecimiento continuo de algunas de las parroquias porque de ello se pueden deducir las consecuentes transformaciones paisajísticas acontecidas. Tan sólo siete parroquias han encadenado tasas de crecimiento positivas en los cuatro periodos intercensales: Noreña; Lugo y Rondiella, en Llanera; La Carrera, Lugones y Pola de Siero, en Siero; San Claudio y Oviedo, en el municipio ovetense. La variación demográfica en períodos intercensales también es útil para determinar 
el grado de crecimiento de las entidades colectivas o parroquias. Así, se constata un fuerte crecimiento entre 1970 y 1981 en las parroquias de Rondiella, 52\%; La Manjoya, 51\%; Lugones, $71 \%$ y Piedramuelle con $51 \%$. En la década posterior los crecimientos superiores al 50\% se localizan en las parroquias de Argüelles, 67\%; Pruvia, 55\% y Viella con un incremento del 67\%. Entre 1991 y 2001 los aumentos más significativos se dieron nuevamente en Pruvia con el 60\% y en Santianes con el 85\%. Finalmente, en la última fase intercensal solamente Pruvia y Viella superaron el 50\%. Estas cifras permiten localizar con bastante precisión aquellos lugares de la comarca en los que la transformación paisajística ha sido más intensa, dado que los crecimientos demográficos intensos vienen, con frecuencia, asociados a cambios socioeconómicos drásticos materializados bien sea en forma de actividades económicas con una alta demanda de mano de obra, un incremento de la función residencial, o una mejora sustancial en la accesibilidad.

Por el contrario, son muchas las entidades colectivas que han sufrido un descenso demográfico continuado entre 1970 y 2011. De tal modo que solo se mencionan aquellas que han experimentado declives más intensos; esto es, superiores al 50\% y que son las parroquias de Palombar, en Ribera de Arriba; Box, Brañes, Caces, Naves, Nora, Pereda, Pintoria, Puerto, Santianes, Trubia y Udrión, en Oviedo; Cayes y Santa Cruz de Llanera, en Llanera, Carbayín, Feleches, La Paranza y Valdesoto, en Siero y la parroquia reguerana de Valduno. Todas ellas situadas en el perímetro comarcal, más rural o minero industrial.

\section{Discusión}

A pesar de que la dinámica reciente de la comarca se caracteriza por un notable crecimiento demográfico, un análisis detallado revela que el número de entidades singulares que pierden población de 400; mayor que el total de núcleos que tienen una tendencia positiva, 113. Esto se traduce en una dinámica que favorece la concentración en núcleos de cierto tamaño y solamente existe dispersión de la población en áreas de alta accesibilidad. Por tanto, en términos superficiales, visto desde una perspectiva espacial predomina el despoblamiento de numerosas entidades homogéneamente repartidas por la comarca, la mayor parte de ellas, de pequeño tamaño que se encuentran en ámbitos de marcado carácter rural o bien afectados por el cese de actividades industriales y mineras. De este modo, los descensos más acusados se localizan en el extremo oriental y meridional de Siero, en las parroquias de Carbayín y Lieres, vinculadas tradicionalmente a la minería; en el valle del Nalón, en el sur del municipio ovetense, entre Olloniego y Tudela Veguín por el mismo motivo; en el entorno de la antigua fábrica ya deaparecida de explosivos de La Manjoya y en el valle del Trubia caracterizado por una fuerte impronta de la industria armera, entre otras. Junto a estas entidades, habría que destacar el intenso proceso de despoblamiento que afecta a los pueblos y aldeas de la comarca de Oviedo lo que ha desencadenado importantes transformaciones paisajísticas debido a la desruralización y al abandono de las actividades propias del sector primario. 
El patrón espacial que dibujan las entidades singulares con incrementos de población muestra el protagonismo de algunos núcleos que ya tenían cierto peso en 1970 como son Pola de Siero, Lugones, Noreña, Posada de Llanera, Colloto, El Berrón o San Claudio, en los que la presencia de equipamientos (sanitarios, educativos, culturales, etc.) y la buena accesibilidad que proporciona la red de infraestructuras ha favorecido la tendencia positiva. Además de estos núcleos, existen otros que actualmente tienen un papel relevante dentro de la comarca y que han experimentado crecimientos muy fuertes debido a que o bien son de nueva construcción o bien han vivido un intenso proceso de transformación; tales son los casos de las urbanizaciones de La Fresneda, Meres y Soto de Llanera, Lugo de Llanera y, sobre todo, La Corredoria que ha pasado de ser un pueblo de 222 habitantes en 1970 a contar con 14.000 en la actualidad, convirtiéndose en uno de los barrios más dinámicos de la capital de Asturias. El resto de entidades con crecimientos demográficos se localizan, en tres ámbitos bien diferenciados; por un lado el eje que forma el surco prelitoral hacia el Este, el que sigue las infraestructuras que comunica Oviedo y Gijón, y finalmente, la franja periurbana más próxima de la capital asturiana.

Si importante es conocer la distribución espacial del fenómeno, no lo es menos su organización temporal. Por este motivo se han establecido las diferentes fases de la dinámica demográfica reciente. Se ha constatado que las fases más intensas en la comarca han sido las que corresponden a los periodos intercensales de 1970 a 1981 y de 2001 a 2011. Simultaneamente, entre 1970 y 2001 se ha producido un intenso retroceso en los municipios de Las Regueras y Ribera de Arriba, las dos vinculadas al valle del Nalón, más rural y afectado por la crisis de la industria tradicional.

\section{Conclusiones}

La dinámica reciente de la población en la comarca de Oviedo se caracteriza por su crecimiento demográfico. No obstante, este crecimiento no es homogéneo y existen claras disparidades dentro del área de estudio. Los análisis de población suelen limitarse a la escala municipal que, en espacios definidos por la difusión urbana resulta insuficiente, puesto que no es posible afinar el fenómeno dentro de unos ámbitos administrativos grandes y heterogéneos. En este artículo se emplean tanto las entidades colectivas o parroquias, que aún se conservan con fines estadísticos en el noroeste peninsular, como las entidades singulares de población que permiten precisar el análisis de los procesos de difusión urbana en los entornos de las ciudades. Gracias a la información que recoge el Nomenclátor se han georreferenciado todas las entidades y, además, mediante la codificación empleada por el INE, se garantiza la coherencia entre los datos actuales, digitalizados, y los publicados en papel con anterioridad al año 2000.

La tendencia global de la población en las últimas décadas muestra un claro aumento de la población urbana, siendo especialmente relevantes el peso de las ciudades de ta- 
maño medio. Este fenómeno ha caracterizado también la historia demográfica española desde mediados del siglo XX, y en Asturias se manifiesta mediante la concentración de la población en su área central. Los municipios que conforma la comarca albergaban al $20 \%$ de la población asturiana en 1970 y en la actualidad ese porcentaje asciende al 28\%, lo que refuerza la hipótesis del aumento de la población urbana a diferentes escalas.

Las entidades que han tenido un crecimiento demográfico positivo dibujan un patrón espacial formando por entidades de la franja periurbana de Oviedo y en los márgenes de los principales ejes que comunican la comarca con el Norte y el Este de Asturias. Los lugares que han sufrido un descenso más acusado del número de habitantes se localizan en zonas de marcado carácter rural y en las entidades afectadas por el cese de actividades mineras e industriales.

En definitiva, el análisis espaciotemporal de la dinámica demográfica de la comarca de Oviedo permite una prospección más fina que las habituales por acercarse a la escala más próxima al poblamiento e identifica las principales fases de la evolución reciente. Espacio y tiempo, por tanto, son las condiciones a priori para futuras investigaciones que vinculen la dinámica reciente de la población y las importantes transformaciones paisajísticas experimentadas en las comarcas asturianas y españolas a partir de 1970.

\section{Agradecimientos}

Quiero mostrar mi agradecimiento a los revisores por sus sugerencias y aportaciones, así como al apoyo financiero prestado por el programa FPU del Ministerio de Educación, Cultura y Deportes (FPU14/04710).

\section{Bibliografía}

Antrop, M., 2004. Landscape change and the urbanization process in Europe. Landscape and urban planning, 67(1-4), pp. 9-26.

Arroyo Pérez, A. (coord.), 2003. Tendencias demográficas durante el siglo XX en España. Madrid: Instituto Nacional de Estadística.

Champion, T., 2001. Urbanization, suburbanization, counterurbanization and reurbanization. Handbook of urban studies, (160), pp. 143-162.

Cheshire, P., 1995. A new phase of urban development in Western Europe? The evidence for the 1980s. Urban Studies, 32(7), pp. 1045-1063.

Criado Hernández, C. y Pérez González, R., 1975. Notas sobre la dinámica y estructura de la población de Asturias (1857-1970). Departamento de Geografía. Universidad de Oviedo. 71 p.

De Cos Guerra, O., 2007. La dinámica metropolitana en España. Análisis estadístico y cartográfico de los municipios a partir de la población y la vivienda. Geographicalia, (51), pp. 59-80. 
Delgado Viñas, C., 1999. Los procesos de difusión urbana en Cantabria. Polígonos, revista de Geografía, (9), pp. 71-96.

Fernández Cuesta, G., 2005. Atlas escolar de Asturias. Ediciones Nobel. 174 págs.

Fernández Cuesta, G., y Quirós Linares, F. (dirs.), 2010,). Atlas temático de España. Ediciones Nobel.

Fernández García, A., 1985. La redistribución de la población en el concejo de Langreo entre 1975 y 1981. Ería, (9), pp. 227-232.

Fernández García, A., 2003. Las pautas del crecimiento urbano posindustrial: de la rururbanización a la ciudad difusa. Ería, (60), pp. 88-92.

Fernández García, A., 2015,. Noreña, un concejo singular. Real Instituto de Estudios Asturianos, 54 páginas.

Fernández García, A., (coord.) 2007. Población, administración y territorio en Asturias. Consejo económico y social del Principado de Asturias, colleción de estudios, Número 6.

Fernández García, F. y Maceda Rubio, A., 1988: Evolución de la población de Asturias entre 1900 y 1981. Análisis parroquial. Ería, (17), pp. 271-276.

Fernández García, F., 1984, El polo de desarrollo de Oviedo. Ería, (7), pp. 135-147.

Fernández García, F. y Herrán Alonso, M., 2013. III. 1.4. El área periurbana de Oviedo. La cuenca del río Nora. En Atlas de los paisajes agrarios de España (pp. 389-398).

Fernández Salinas, V., 2001, Las tendencias demográficas recientes de la población andaluza. Ería, (54/55), pp. 77-93.

Fernández Soto, M., 2014. Las transformaciones territoriales y paisajísticas recientes de la comarca de Avilés. Tesis doctoral, Universidad de Oviedo.

García Fernández, J., 1975. La organización del espacio y economía rural en la España atlántica. Siglo veintiuno de España ed.

Herrán Alonso, M., 2002. La evolución del paisaje en un espacio periurbano: Lugones-Posada de Llanera (Asturias). Ería, (57), pp. 25-48.

Herrán Alonso, M., 2008. La producción de espacio residencial de baja densidad en el municipio de Oviedo (1957-2003). Tesis doctoral. Universidad de Oviedo.

Indovina, F. (ed.), 1990. La città diffusa. Venecia. DAEST.

Klaassen, L.H., Molle, W.T.M., Paelinck, J.H.P. (eds.), 1981. Dynamics of Urban Development. Aldershot, Gower.

López Fernández, B., 1988. La gradación del envejecimiento en los espacios intraurbanos. Estructuras demográficas y crecimiento urbano en Gijón. Eria, (17), pp. 201-233.

López Fernández, B., 1992, La población de Asturias. En Geografía de Asturias, pp. 81-96.

López Fernández, B., Fernández Cuesta, G., Fernández García, F., y Fernández Prieto, J.R., 1994. Atlas social de las mujeres asturianas. Oviedo: Servicio de Publicaciones del Principado de Asturias.

López Fernández B., 2010. La población. En Atlas temático de España, vol. 2, pp. 9-155. 
López Fernández, B., 2016. Poblamiento y declive demográfico en Asturias, 2000-2014. Ería, (99100), pp. 95-108.

Morales Matos, G., 1982. El intercensal 1970 - 1981 en Asturias. Ería, (3), pp. 82-87.

Morales Matos, G. (dir.), 1992. Geografía de Asturias. Editorial Prensa Asturiana. 4 tomos.

Morán Alonso, N., Obeso Muñiz, Í, Hernández Aja, A., y Fernández García, F., 2017. Challenges for the revitalisation of peri-urban agriculture in Spain: Territorial analysis of the Madrid and Oviedo metropolitan areas. Moravian Geographical Reports, 25(3), pp. 192-207.

Obeso Muñiz, Í., y Cortizo Álvarez, T., 2016. Los cambios de residencia en el concejo de Oviedo, 1996-2004. Ería, (99-100), pp. 325-337.

Pérez González, R., Fernández García, F. y Morales Matos, G., 1983, La población y el poblamiento en Quirós Linares, F. (dir.): Geografía de Asturias, tomo IV. Editorial Ayalga. Salinas, pp. 234-269.

Pozo Rivera, E., y Rodríguez Moya, J., 2006. Transformaciones sociodemográficas recientes en las comarcas Castellano Manchegas limítrofes con la Comunidad de Madrid. Anales de geografia de la Universidad Complutense, (26), pp. 249-281

Puyol, R., 1999. Dinámica de la población en España: cambios demográficos en el último cuarto del siglo XX. Síntesis.

Quirós Linares, F., 1982. La delimitación territorial de las parroquias asturianas. Ería, (3), pp. 79-82.

Quirós Linares, F., 1993. División Parroquial de Asturias. Servicio Central de Publicaciones del Principado de Asturias, 78 pp., 1 lám.

UN, 2014. World Urbanization Prospects: The 2014 Revision-Highlights. UN.

UN, 2013.World Population Prospects: The Revision 2012. Volume 1, Comprehensive Tables (United Nations publications ST/ESA/SER.A/336).

Van den Berg, L., Drewett, R., Klaasen, L. H., Rossi, A., \& Vijverberg, C. H., 1982. Urban Europe: A study of growth and decline. Elmsford, N.Y./Oxford, England, Pergamon Press, 1982, xxii, 162 p. (Urban Europe Vol. 1). 